\title{
AccessScope project: Accessible light microscope for users with upper limb mobility or visual impairments
}

\author{
AWAIS MANSOOR ${ }^{1}$, WAMIQ M. AHMED ${ }^{1}$, ALA SAMARAPUNGAVAN ${ }^{2}$, JOHN CIRILLO ${ }^{3}$, \\ DAVID SCHWARTE ${ }^{4}$, J. PAUL ROBINSON ${ }^{1}$ \& BRADLEY S. DUERSTOCK ${ }^{3}$ \\ ${ }^{1}$ Department of Basic Medical Sciences, School of Veterinary Medicine, Purdue University, West Lafayette, Indiana, USA, \\ ${ }^{2}$ Department of Educational Studies, College of Education, Purdue University, West Lafayette, Indiana, USA, ${ }^{3}$ Center for \\ Paralysis Research, School of Veterinary Medicine, Purdue University, West Lafayette, Indiana, USA, and ${ }^{4}$ Assistive \\ Technology Center, Teaching and Learning Technologies, Purdue University, West Lafayette, IN, USA
}

\begin{abstract}
Purpose. A web-based application was developed to remotely view slide specimens and control all functions of a researchlevel light microscopy workstation, called AccessScope. Students and scientists with upper limb mobility and visual impairments are often unable to use a light microscope by themselves and must depend on others in its operation.

Method. Users with upper limb mobility impairments and low vision were recruited to assist in the design process of the AccessScope personal computer (PC) user interface. Participants with these disabilities were evaluated in their ability to use AccessScope to perform microscopical tasks. AccessScope usage was compared with inspecting prescanned slide images by grading participants' identification and understanding of histological features and knowledge of microscope operation.

Results. With AccessScope subjects were able to independently perform common light microscopy functions through an Internet browser by employing different PC pointing devices or accessibility software according to individual abilities. Subjects answered more histology and microscope usage questions correctly after first participating in an AccessScope test session.

Conclusions. AccessScope allowed users with upper limb or visual impairments to successfully perform light microscopy without assistance. This unprecedented capability is crucial for students and scientists with disabilities to perform laboratory coursework or microscope-based research and pursue science, technology, engineering, and mathematics fields.
\end{abstract}

Keywords: Accessible light microscope, remote microscopy, students with mobility impairments, students with visual impairments, science education

\section{Introduction}

This study represented a major evolution of our accessible light microscope, called AccessScope, with the development of a novel control software application usable by persons with severe disabilities. Previously, AccessScope was developed for students and scientists with upper limb mobility impairments to control all aspects of a research-level light microscope independently. Once loaded with up to 50 slides, users with motor impairments were able to perform common light microscopical functions and image capturing without assistance [1]. Further investigations showed that AccessScope would also be useful for users with low vision [2].

Our AccessScope control software applications were developed specifically for users with upper limb mobility and visual impairments, which represented a significant advantage over current commerciallyavailable software for controlling a light microscope from a local personal computer (PC) [1,2]. The graphical user interfaces (GUIs) of both a local and web-based AccessScope applications were designed from the input of experts and participants with a wide range of disabilities. Users were able to make changes to the PC environment to suit their 
individual abilities and preferences by employing different input devices and assistive technology (AT) software and hardware. The most recent version of the AccessScope application was developed to be remotely operated through a standard Internet web browser. This flexibility allows users to perform light microscopy from home, a laboratory classroom or anywhere there is Internet access on their own time.

The light microscope is one of the most common laboratory tools used by science students and scientists. Unfortunately, students with severe physical disabilities have tremendous difficulty using a conventional light microscope by themselves [1-4]. AccessScope allows students with mobility and visual impairments for the first time, independent microscopical usage, the ability to perform independent research and active learning experiences with light microscopy.

Traditionally, students with disabilities have relied upon classmates without disabilities or lab assistants when using a light microscope during laboratory class $[3,5,6]$. With AccessScope, students with disabilities do not have to be dependent on others and have the freedom to use a light microscope on their own schedule, not only during class times. This independence is also necessary to conduct individual research activities. AccessScope allows researchers to perform brightfield and fluorescent microscopical viewing and subsequently manage captured micrographs with other software applications for image analysis, morphometry or three-dimensional histological visualisation.

Performing hands-on experimental techniques and exploring with a light microscope provides active learning experiences critical for more in-depth understanding of microscopical imaging and scientific concepts $[7,8]$. Activity-based learning is a standard of teaching science at all educational levels for courses, including histology, cell biology, botany, microbiology and materials science. It is believed that the ability to actively interact with scientific concepts and actual practices during laboratory classes in science, technology, engineering, and mathematics (STEM) courses improves the overall learning experiences of students and results in better test scores [9].

AccessScope allows students with disabilities to perform light microscopy during a laboratory-based class along side their classmates. We believe such accessible laboratory tools are essential for promoting greater inclusion of students with disabilities in science courses $[3,5,10]$. This is particularly important during secondary, postsecondary and graduate education where significant amounts of laboratory practice and equipment usage are required $[11,12]$. Only $0.3 \%$ of graduate students that earned a doctorate degree in science or engineering had a mobility or visual impairment [13], and only $2 \%$ of employed scientists and engineers 35 or younger had a disability [14]. Thus, there is a tremendous need to assist students with disabilities to successfully continue to pursue STEM fields throughout postsecondary and graduate education to the work force.

\section{Methods}

\section{AccessScope workstation}

A Dell ${ }^{\circledR} \mathrm{PC}$ with an Intel ${ }^{\mathbb{R}}$ Core $^{\mathrm{TM}} 2$ Duo processor controls the major hardware components of the AccessScope workstation and displays the microscope images of slide specimens to its computer monitor [Figure 1(a)]. Chief among these components is an Olympus ${ }^{\circledR}$ BX61 ${ }^{\circledR}$ (Center Valley, PA) research light microscope with motorised objective nosepiece, condenser, filter wheel, illuminator and $\mathrm{Z}$ focus drive [Figure 1(b)], which was connected via a RS232C cable. A digital camera was mounted to the ocular portion of the microscope [Figure 1(c)], and a motorised stage by $\mathrm{Ludl}^{\circledR}$ Electronics Products (Hawthorne, NY) that moves in $X$ and $Y$ axes [Figure 1(d)] was attached. Integrated with the stage was a bulk slide autoloader that mechanically loaded up to fifty slides onto the stage [Figure 1(e)]. A camera theta rotator was developed to rotate the

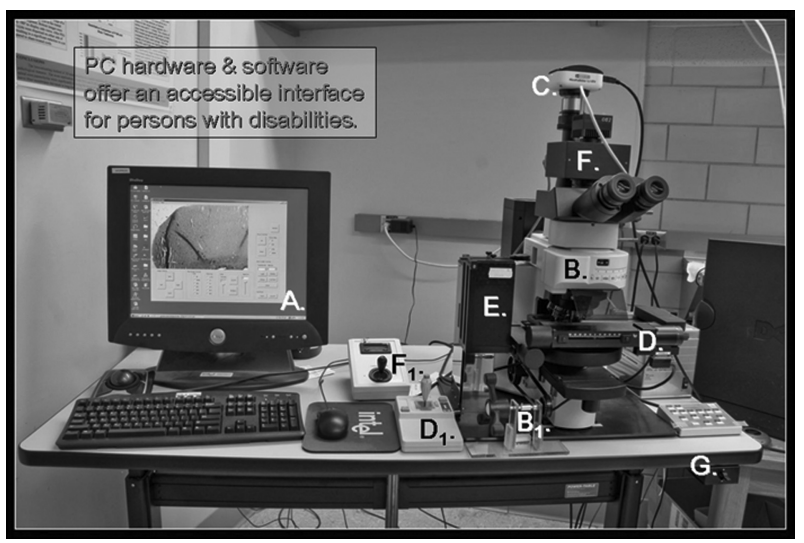

Figure 1. Running the web-based AccessScope application through a PC interface (a), individuals with either hand and arm impairments or low vision could remotely operate all microscopical functions (b) of the system and view the slide specimen (c) by themselves. Only loading slides into the 50 -slide autoloader would require human assistance (e). In addition to the remote control of AccessScope through the Internet, redundant hardware controls were adapted for local users with impairments. A joystick $\left(\mathrm{d}_{1}\right)$ was provided to alternately move the stage (d) in X and $\mathrm{Y}$ directions. $\left(\mathrm{f}_{1}\right) \mathrm{A}$ joystick controlled the theta rotation of the camera rotator (f). By depressing a button on top of the joysticks would accelerate movement. Most participants, except those with severe hand motor deficits, were able to use these joysticks. AccessScope was placed on a mechanically-adjustable height table to accommodate the different seating heights of local users. 
digital camera to theta position the real-time microscope image for enhanced viewing [Figure 1(f)]. The entire system rested upon an automatic heightadjustable worktable, which could be raised or lowered by a two-way momentary, toggle switch according to participants' seating height needs [Figure 1(g)]. Subjects used an office chair or manual or motorised wheelchair when operating the AccessScope workstation, which all differed in height.

AccessScope is operated through a PC interface that controls all features of the microscope and other components. The PC was adaptable to accommodate different user's preferences and disability by employing different pointing devices and AT software [15]. The PC ran Windows ${ }^{\circledR}$ operating system to facilitate the use of third-party commercial AT software to reduce costs and to readily accommodate participant users during trials. AccessScope was demonstrated to be compliant with ZoomText ${ }^{\mathbb{R}}$ (Ai Squared ${ }^{\mathbb{R}}$, USA) screen magnifier, JAWS ${ }^{\mathrm{TM}}$ (Freedom Scientific ${ }^{\mathbb{}}$, USA) text screen reader and Microsoft ${ }^{\mathbb{R}}$ accessibility programmes, StickyKeys ${ }^{\mathrm{TM}}$ and MouseKeys ${ }^{\mathrm{TM}}$.

A variety of PC input devices including mouse, trackball, keyboard and speech recognition was made available to each participant prior to testing. However, our AccessScope applications were designed to be entirely controlled by a keyboard, as keyboarding was found to be the most universal method for controlling a PC by our target groups $[1,2]$. A standalone application was first developed to evaluate the usability of its GUI by our target group, specifically for users with hand and arm mobility and visual impairments. This AccessScope application was programmed in $\mathrm{C}++/$ Visual $\mathrm{C}++$ to run on Microsoft ${ }^{\circledR}$ Windows $2000^{\circledR}$.

The GUI of this standalone AccessScope application later served as the basis for a web-based application that was also programmed in $\mathrm{C}++$ / Visual $\mathrm{C}++$. The web-based AccessScope application was remotely operable through Internet $\mathrm{Ex}^{-}$ plorer $^{\circledR}$ (Figure 2). No installation of the AccessScope application was necessary except for the remote user to run ActiveX ${ }^{\mathrm{TM}}$ control, which is downloadable from its web site. Active $\mathrm{X}^{\mathrm{TM}}$ enabled a client to remotely control the AccessScope through the server, including a high-resolution 1394-based QImaging ${ }^{\circledR}$ (Surrey, BC, Canada) camera that acquired images from the microscope. Captured images were transmitted as a live stream to the client's PC using state-of-the-art FFMPEG streaming library. To ensure portability to different platforms, the live stream was encoded in MPEG format to enable viewing on standard video client applications, such as Windows Media Player ${ }^{\circledR}$, VLC $^{\circledR}$ or RealPlayer ${ }^{\circledR}$ (Figure 3).

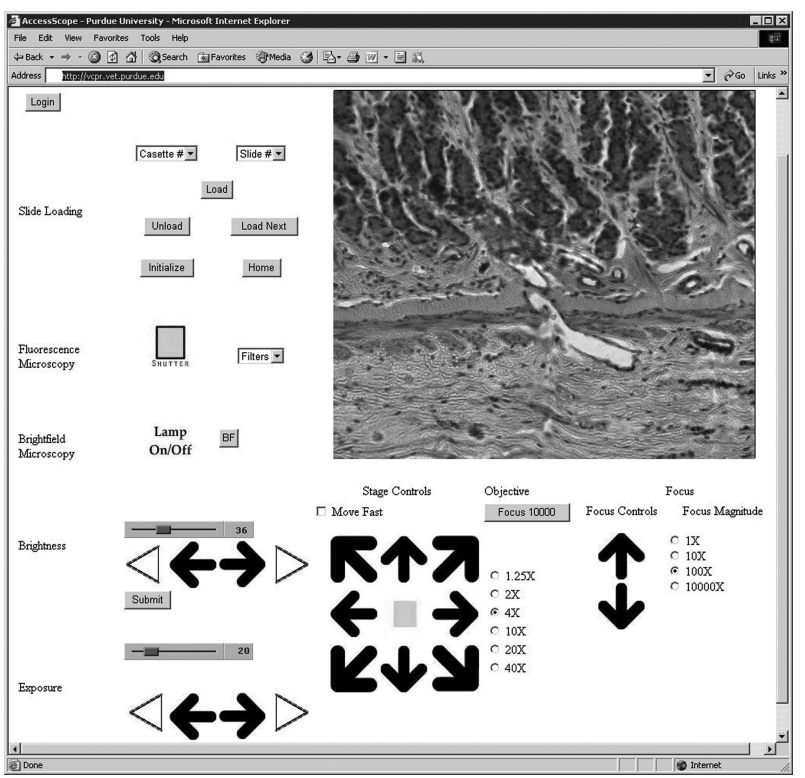

Figure 2. The web-based AccessScope program was assessed using Microsoft ${ }^{\mathbb{R}}$ Internet Explorer. The design of the GUI was based on participant and expert usability testing. The controls wrap around the preview window from the top left to the bottom right to facilitate computerised screen reading and tabbing with a keyboard. The arrangement of the controls was based on the order of typical microscope operation. Each button had an XHTML alt tag that described its function for screen reading and listed its hotkey function for immediate keyboard execution. Continuous functions such as stage movement, focussing, brightness and exposure could be adjusted by different methods; increasing steps by multiples, automatically traveling to extremes with arrow buttons or by numerical entry. Default settings for brightfield microscopy were defined to enable fine adjustments rather than requiring gross changes be made each time AccessScope is used. Changes to the GUI controls could be made to accommodate individual needs.

\section{Participant recruitment and evaluation}

Ten participants with a variety of upper limb mobility or visual impairments were used during the design and assessment portions of this study (Table I). Prospective participants were notified of our study by Purdue University's Disability Resource Center on our behalf to ensure subject anonymity. If interested, these individuals would contact us on their own volition. Participants had to be 18 years or older with a permanent upper limb mobility impairment or visual impairment that was non-correctable with glasses, but not totally blind.

The design of the AccessScope GUI was initially accomplished using a heuristic approach involving three student users with motor or visual disabilities and two experts in software AT with low vision and an upper limb mobility impairment. User assessments regarding the design of the application's GUI were gathered when evaluating the standalone application. These changes were incorporated into 

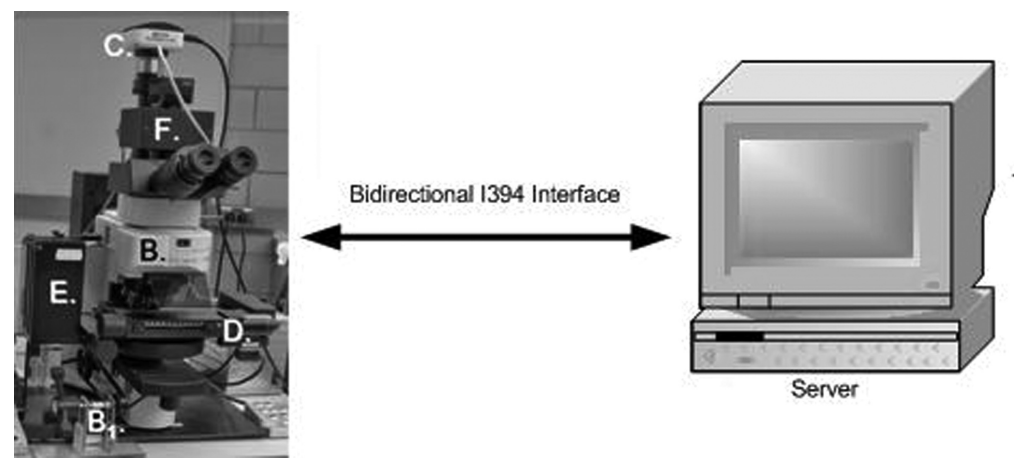

Bidirectional TCP Connection for Scope

Control

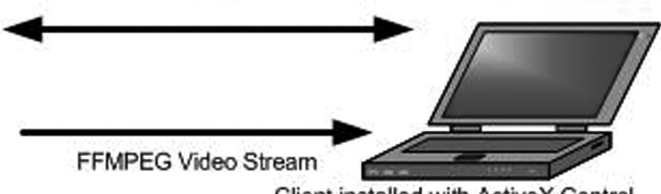

Client installed with ActiveX Control

Figure 3. The complete network interface of the AccessScope system is shown. The microscope equipment was attached to the server via an IEEE1394 serial bus interface for high-speed communications and real-time data transfer. The machine labeled Server hosted specific server applications to perform various interface operations of the software among the host, client and microscope. The FFMPEG video encoding library in the Server encoded each frame using an MPEG-4 codec and streamed the data through FFSERVER. The server also hosted the Apache server for the real-time web interface of the software. Another $\mathrm{C} / \mathrm{C}++$ server application on the server machine formed a TCP/IP connection to interact with the ActiveX control installed on the client PC that remotely controlled the microscope equipment.

Table I. Description of participants that evaluated AccessScope.

\begin{tabular}{lclll}
\hline Participants & Gender & Age & Disability & Major deficits \\
\hline 1 & F & 45 & Eye injury & Lacks visual acuity \\
2 & F & 21 & Albinism & Reduced visual acuity, nystagmus, astigmatism \\
3 & M & 30 & Progressive myoclonic epilepsy & Muscle weakness, intention tremors, ataxia \\
4 & $\mathrm{M}$ & 22 & Vision loss due to craniotomy & Vision loss in upper portion of the visual field \\
5 & $\mathrm{~F}$ & 51 & Amblyopia & Lacks depth perception, poor 3D spatial acuity \\
6 & $\mathrm{M}$ & 23 & Charcot-Marie-Tooth disease & Poor fine motor control in hands and fingers \\
7 & $\mathrm{M}$ & 39 & Retinitis pigmentosa & Tunnel vision, poor visual acuity \\
8 & $\mathrm{M}$ & 43 & C-6 spinal cord injury & Lacks fine motor skills in hands and fingers \\
9 & $\mathrm{M}$ & 23 & Retinitis pigmentosa & Poor light sensitivity, reduced visual field \\
10 & $\mathrm{M}$ & 21 & Juvenile macular degeneration & Poor visual acuity, low central vision \\
\hline
\end{tabular}

the web-based application for larger participant trials that involved both usability and performance-driven testing.

A combination of qualitative and quantitative assessments was used to evaluate the usability and effectiveness of AccessScope. Participants were asked to comment on the usability of AccessScope when exploring a slide containing a histological section of stomach. While personally manipulating the software controls of the system, participants were verbally instructed on how to operate AccessScope and the function of each command. Subjects were requested to identify aspects of AccessScope that they thought were beneficial or unfavourable. They were also asked to compare using AccessScope to any previous experiences they have had with conventional microscopical methods, including studying databases of slide images or histology atlases.

A repeated measures design was employed in which eight participants served as their own controls to rate their performance on a quiz on basic histology and microscopy concepts. The quiz was designed to evaluate their recall of the histological specimen and reasoning skills in determining its function. The quiz consisted of six multiple-choice questions, which was preceded by a two-paragraph long narrative of histology that described the four different tissue types of the body and the relationship between cell shape and function (Appendix 1).

To control for practice effects, half of the participants either first explored a slide with a cross-section of stomach using AccessScope or studied a micrograph of a blood smear slide using a graphics program. During a second session, the participants would take the other test. The identity of the slide specimens were not disclosed to subjects. Test subjects were able to study each slide as long as they wished before taking the same six question quiz. Test scores were statistically compared between participants taking the AccessScope tests and digital slide tests during the first or second sessions. $p<0.05$ was considered statistically significant.

\section{Results}

\section{GUI design and development}

With the aid of student participants, investigators were able to identify universal design features for the 
AccessScope application's GUI usable specifically for persons with visual or upper limb mobility impairments (Figure 2). PC users with visual impairments predominately employed a keyboard to activate graphical commands. Although some users with upper limb mobility impairments were proficient using a mouse or trackball, keyboard usage was also very high among these individuals for activating commands and precise maneuvering of the pointer (Table II). Thus, all commands of the AccessScope application were made to be operable through keystrokes as well as point and clicking. Users were able to navigate through the groups of controls by pressing 'tab' and arrow keys. Activation of these controls was achieved by pressing 'enter', the spacebar or left-mouse click. Combinations of defined keystrokes or 'hot' keys were also programmed for each command of the AccessScope application. For example, pressing 'alt- $4,6,2$ or 8 ' automatically moved the stage to the left, right, forward or backward, respectively. Alternate attributes or 'alt tags' were provided for each graphical button to describe its function and its hot key. These alt tags were recited by JAWS ${ }^{\circledR}$ screen reader and displayed when the pointer was positioned over the command.

Another GUI design feature that we incorporated into the application was automatic and default settings. Autoexposure is a common feature that decreased the need to manually adjust the exposure rate when performing microscope functions, like changing the objective. This automatic feature often eliminated extra steps for the user. However, exposure rate and other settings could be manually modified by the user. By using default settings for exposure, brightness, brightfield imaging, magnification and focus, users would be able to automatically discern specimens in the preview windows when slides were loaded onto the stage. Without this feature, novice users would be unsure what step to perform next if the AccessScope preview window showed total blackness after loading a slide. However, if the image was simply a little too bright or dark or out of focus then users would be more confident on what to do next to get a clearer view of the slide

Table II. Assistive technology and input devices employed by participants to control the web-based AccessScope user interface.

\begin{tabular}{lc}
\hline AT used for AccessScope operation & No. of participants \\
\hline Keyboard/AT keyboard & 10 \\
Mouse/trackball & 7 \\
Multiple, large-sized PC monitors; & 5 \\
$\quad$ reduced screen resolution; & \\
$\quad$ computer glasses & 3 \\
JAWS & \\
ZoomText & screen reader \\
\end{tabular}

specimen. By requiring users to make fine adjustments to microscope views of specimens provides a learning experience for students and reinforces the idea that they are operating a real-light microscope rather than simply receiving pre-focussed, captured histological images. We believe this promotes an active learning experience for students on how a light microscope works (see Conclusions).

The order of the AccessScope commands in the GUI from top to bottom and then from left to right was chosen to predict users' actions (Figure 2). During light microscopical viewing certain procedures can be anticipated to occur sequentially. By anticipating user's actions usability could be greatly improved. For instance, once brightfield or fluorescence viewing was selected, we expected that brightness and exposure would likely need to be adjusted followed by moving the stage. For low vision users, this order of controls resulted in more efficient selection of subsequent commands because they often employed tabbing to progress through commands.

Viewing slide specimens through the application window instead of through the eyepieces [Figures 1(a) and 2] overcame a major physical obstacle for both users with motor and visual disabilities. Because of the orientation of the eyepieces, wheelchair users have difficulty peering into a conventional upright microscope. During the design phase of AccessScope all of our evaluators with visual impairments stated that they had difficulty seeing through binocular eyepieces.

\section{Participant usability assessment}

During slide exploration with the web-based AccessScope application, 10 participants described what design features or functions were beneficial or disadvantageous for them. Seven individuals with visual impairments and three people with mobility impairments evaluated the usability of AccessScope by employing a variety of PC input devices and AT (Figure 4). All participants used the keyboard when using AccessScope. In addition, many also preferred to use a mouse, especially users with mobility impairments. Some participants with less visual deficits also used a mouse in concert with screen magnification. Subjects with profound vision loss relied on keyboarding and JAWS ${ }^{\circledR}$ screen reader to operate AccessScope. The majority of participants with visual impairments employed a keyboard and enlarged the AccessScope display either with larger monitors, reducing screen resolution or screen magnifying software (Table II).

All participants found that independent operation was the greatest benefit of AccessScope. Over half of 

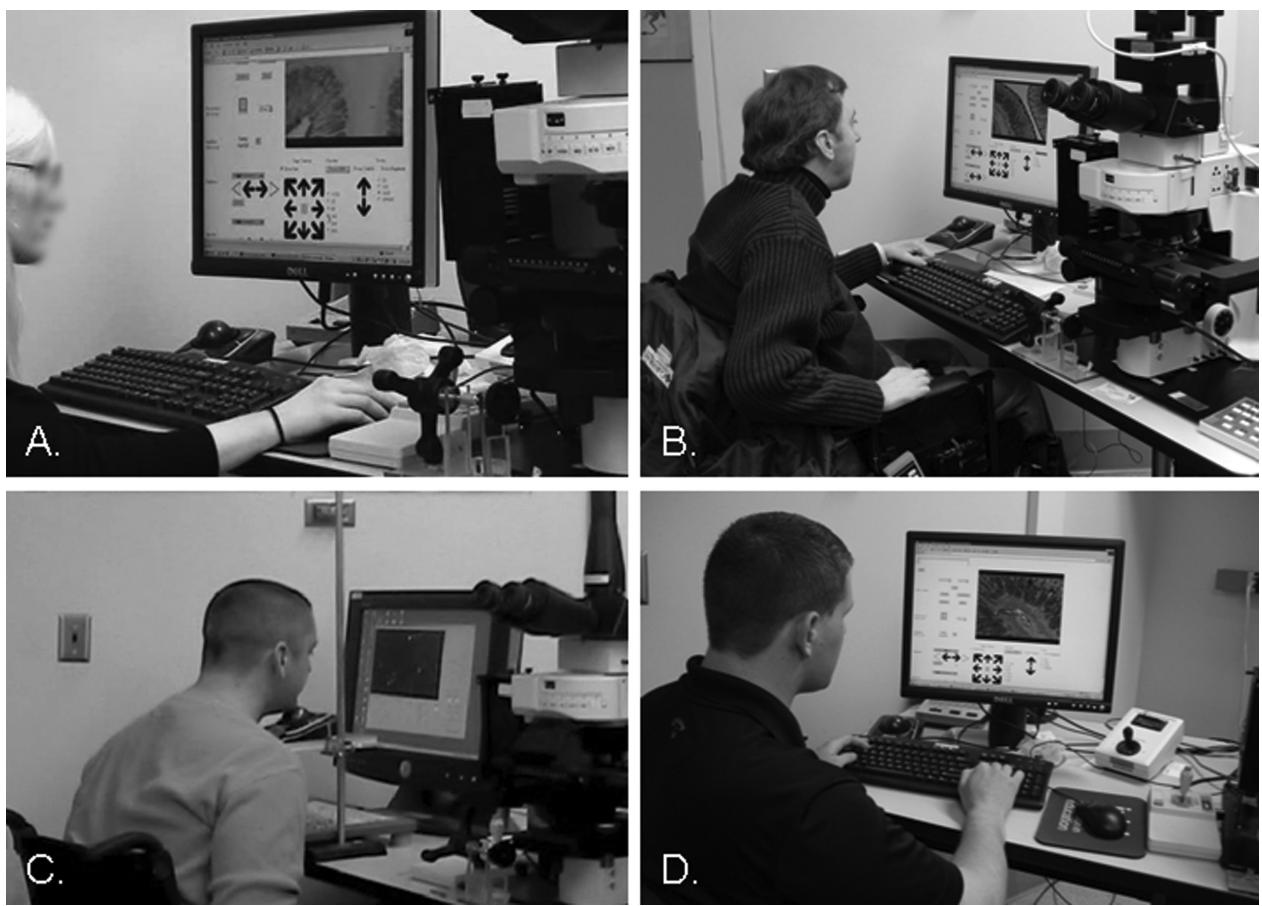

Figure 4. Participants that tested AccessScope used different methods to operate the PC interface. (a) A subject with low vision and nystagmus preferred to use the preview window for microscopical viewing than the binocular microscope eyepieces. Her sight was sufficient to use a mouse to control AccessScope. The screen was magnified to better view the GUI. (b) A quadriplegic with limited arm function but no hand movement employed both mouse and keyboard. (c) Another quadriplegic with intention tremors had difficulty selecting objects by hand. He used a programmable keyboard with oversized keys and moved the pointer with his chin using an elevated trackball. (d) A subject with profound low vision cannot read the GUI commands so uses JAWS to speak the commands to him. He has adequate vision to discriminate shapes and colors of histological features of interest in AccessScope's preview window.

the students had never used a light microscope before by themselves. All subjects found that the graphical buttons and controls were not only, more accessible to them than traditional microscope controls, but also more comfortable because of their familiarity with similar GUIs in other software applications. The single-window view of all the AccessScope controls presented the pertinent microscope commands to first-time users at once, instead of introducing groups of controls at different times. Participants were able to quickly learn what commands they were to perform and in what succession.

All participants with either visual or mobility impairments responded that they liked viewing through the preview window on the PC monitor. Evaluators that relied primarily on one eye for vision were unable to adequately use binocular microscope eyepieces. Two other participants with a nystagmus prevented them from fixating on the eyepieces' field of view (FOV). The AccessScope monitor permitted them to repeatedly scan the preview window until they perceived the entire FOV. All participants with mobility impairments appreciated the ability to observe the specimen through the preview window while simultaneously adjusting microscope settings. With conventional microscopy these users could only peer through the eyepieces or operate its controls if it was repositioned correctly for them.

The most common drawback of AccessScope that participants stated was a slow response time to users' commands, which was encountered when using the web-based AccessScope application but not the standalone programme. Real-time responsiveness to users' actions is necessary to detect subtle changes to the preview image, such as during focusing and adjusting brightness. Also, additional auditory and visual feedback when initiating GUI commands and after completion of the function was requested by all participants. Often both users with motor or visual impairments would activate graphical controls tenuously. Confirmation that a command was executed was needed. In addition, for commands that are not immediately performed, time-lapse and current status indicators were necessary to inform users not to repeatedly execute a command until it was first completed.

\section{Participant performance testing}

Eight participants took a short multiple-choice quiz after reviewing a slide with AccessScope or a digital 
scan of a slide at $40 \times$ magnification during two separate sessions. The quiz questions were the same between both tests with a statement regarding the relationship between cell shape and its function (Appendix 1). Half of the participants evaluated a slide with a section of stomach with AccessScope during the first testing session and evaluated a digital scan of a blood smear during the second testing session. The other half participated in the sessions in reverse order. In Figure 5, the subjects in Group 1, which took the AccessScope test first and the digital slide test second, averaged 5.5 correct answers for both tests combined. The other four participants in Group 2, which took the Digital slide test first and the AccessScope test second averaged 5.75 correct answers (Figure 5). However, Group 1 did significantly better on the Digital slide test than on the AccessScope test ( $p=0.015$, paired $t$-test) (Figure 5). The Digital slide and AccessScope test scores for Group 2 were not significantly different $(p=0.84$, paired $t$-test) (Figure 5). There were no statistical significant difference in test scores between Groups 1 and 2 for either the AccessScope test (1.5 vs. 2.75, despite the large numerical difference) or the Digital slide test $(4.0$ vs. 3.0) $(p>0.05$, unpaired $t$-test) (Figure 5).

The overall average AccessScope test score for all eight participants was 2.125 compared to the average Digital slide test score of 3.5 , which were signifi-

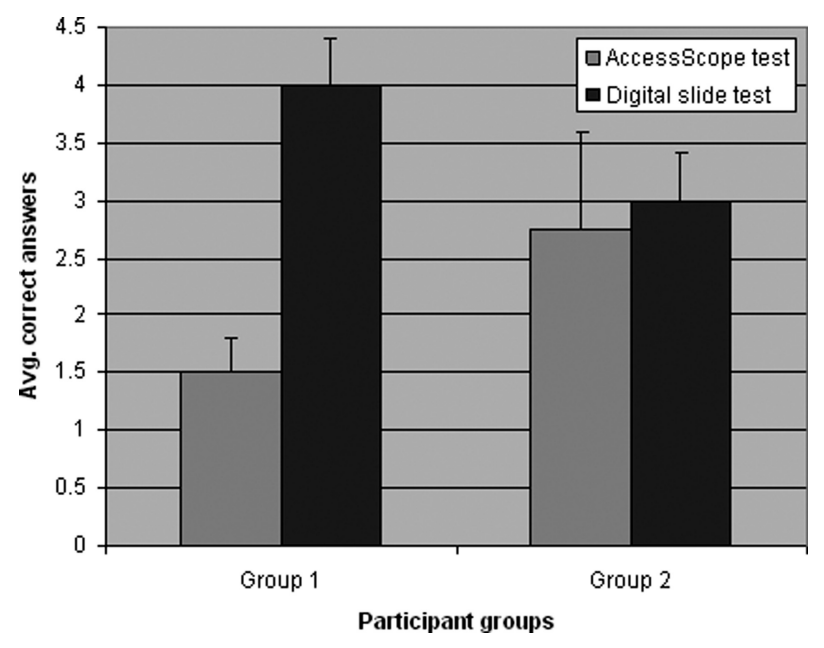

Figure 5. Test scores by participants that took either the AccessScope test or Digital slide test during the first or second session were averaged and statistically compared (standard error bars are shown). In Group $1(n=4)$, there was a significant difference in test scores between the AccessScope test during the first session and the Digital slide test during the second session. In Group $2(n=4)$, there was no significant difference between the Digital slide test score received during the first session and the AccessScope test score earned during the second session. The sums of both test scores were statistically similar between Groups 1 (5.5) and 2 (5.75). Likewise for either test, there were no significant differences in scores between both Groups. cantly different ( $p=0.033$, unpaired $t$-test). Thus, the average score from the AccessScope test during the first session (Group 1) was considerably lower than the average scores from the other three testing sessions.

Figure 6 showed how many subjects answered each test question correctly. The number of correct answers was compared whether the AccessScope or Digital slide tests were taken during the first or second sessions. Questions 1 and 2 referred to characterising the tissue samples based largely on observation at low magnification (Appendix 1). Group 2 did better on both tests than Group 1 (Figure 6).

Questions 3 and 4 showed the greatest disparity in being correctly answered between AccessScope and Digital slide test takers. More than twice as many Digital slide test takers answered correctly for questions 3 and 4 compared to AccessScope test takers (Figure 6). Question 3 required participants to determine cell shape at high magnification. Question 4 required subjects to infer the identity of the samples based on their morphology and a short description about histology that was provided. The correct answer to question 5 relied upon correctly deducing the previous questions (Appendix 1). For both groups test scores were higher after the second session (Figure 6).

Question 6 related to basic light microscope operation and not to the histological specimen (Appendix 1). Subjects scored slightly higher on this question when taking the AccessScope test (6 of 8

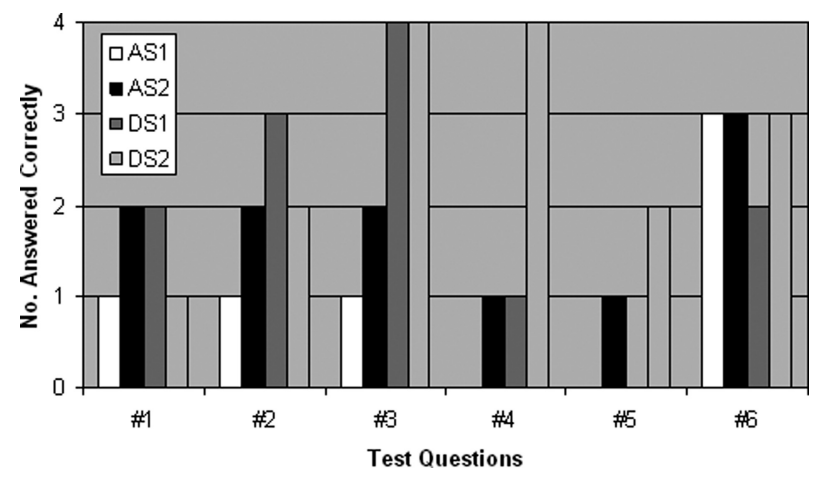

Figure 6. A distribution of how many participants answered the six multiple-choice questions correctly during AccessScope tests during first (AS1) and second sessions (AS2) and Digital slide tests during first (DS1) and second sessions (DS2). Group 1 correct answers are represented by the middle two bars for each question. Group 2 correct answers are shown by the outer bars. For questions 1 and 2, Group 2 did better on both tests. For questions 3 and 4, both groups scored better on the Digital slide tests. Scores for questions 5 were higher after the second session. Question 6, regarding microscope operation, was answered more correctly by subjects who had already performed the AccessScope session. Overall, Digital slide test takers averaged statistically higher scores than AccessScope test takers. 
participants answered correctly) then after taking the Digital slide test (5 of 8 correct answers). The lowest number of correct answers for question 6 occurred for subjects that had taken the Digital slide test first and had not used AccessScope yet (Figure 6).

\section{Conclusions}

In this study, we developed and tested a new, webbased AccessScope control application using participants with a range of upper limb mobility or visual impairments. Prospective AccessScope users include secondary, postsecondary or graduate students with physical or sensory disabilities as well as scientists and engineers that become impaired due to a late onset disorder, trauma or repetitive stress injury.

The student participants were surveyed during AccessScope studies regarding their attitudes toward the hands-on nature of postsecondary laboratory courses in STEM fields. Specifically, we asked, 'Has the prospect of knowing that a course requires using a microscope or other laboratory equipment discouraged you from wanting to take that class? If yes, what did you end up doing?' Every participant with a disability, which affected performing a daily living activity, stated they purposely avoided courses with laboratory requirements. They decided to take theory-based courses that would fulfill their college science requirements or chose in advance not to pursue a STEM major.

Participants were also queried, 'Has the thought or realisation of having to use hands-on equipment discouraged you from pursing a field of study or occupation? If so, what was the study or job?' Again, most responded they felt the physical obstacles likely to be encountered in STEM education were too daunting or impossible to overcome. Those students that were interested in STEM fields had chosen related, less physically demanding majors. For example, one participant wanted to study medicine but decided to major in psychology. Another student subject had aspirations to be a veterinarian but decided to study agricultural business. Therefore, we believe providing accessible tools or techniques that enables students with disabilities to perform laboratory tasks is imperative for them to actively participate in STEM fields and ultimately pursue STEM careers.

\section{Usability study}

Participants were able to successfully perform common light microscope activities by themselves and locate general histological structures in the preview window of the AccessScope system. During this usability assessment, we found some issues that could be improved with AccessScope. One major problem with the AccessScope application that participants encountered was decreased responsiveness or a delay in refreshing the image after command execution. This reduced responsiveness was likely due in part to the high resolution video that was transmitted from AccessScope's camera. To display a high-resolution video stream to the PC from the AccessScope camera at normal frame rate within normal bandwidth parameters, the FOV of video transmission had to be reduced. We will make additional adjustments to the video transmission to improve AccessScope refresh rates.

Another point of issue that was common with subjects' assessments was a need for greater feedback from the controls during AccessScope operation. Accessible controls to perform microscopical functions were mandatory for independent operation; however, subjects requested more dynamic feedback from the AccessScope controls. Possibilities included visual or auditory cues when commands are initiated, status bars or icons to indicate the processing of a command after execution, and the current status of the system components. Because of their disabilities, participants were sometimes tentative when activating a control. If there was a lag in response, participants were additionally unsure if they needed to repeat a command. Therefore, some type of feedback would alleviate their uncertainty whether a command was executed.

During testing subjects also found that navigating the slide through the AccessScope preview window tedious. Finding a specific region of interest on a slide, which often contains several tissue sections, requires visually scanning through a relatively small FOV that becomes smaller at higher magnifications. This problem is universal and not restricted to users with disabilities. A solution to this problem is to initially scan the entire slide and create a lowresolution, dynamic image map of the slide. The relative position of the microscopist's current FOV would be automatically tracked and displayed in realtime on the slide image map. Each loaded slide would undergo this procedure.

\section{Performance study}

Participants were tested in their ability to learn histology with AccessScope compared to a traditional method of studying a pre-scanned image of a histological section. Because of the low number of participants, we employed a repeated measures design for each participant to be their own control. In two groups, participants performed either the AccessScope or Digital slide session first or second 
to reduce practice bias. The combined scores of both tests were almost identical for each group. This shows that the test taking ability of the participants from both groups were very similar. Although all participants were undergraduate students or had a postsecondary degree, none of the subjects majored in science or were scientists.

The group that took the AccessScope test first and the Digital slide test second did significantly better on the Digital slide test, but the other group scored approximately the same on both tests. However, when all participants were combined, they averaged higher scores on the Digital slide test. The major factor for averaging overall lower AccessScope test scores was due to taking the AccessScope test during the first session. The test scores from the other three sessions were similar. We believe that practice did have an effect on second session testing. The inspection of Digital slides was much simpler for individuals who mostly never used a microscope before. Subjects practiced on the AccessScope system for approximately 15-30 min before conducting the histology performance test. We believe performing the Digital slide session first helped students prepare how to use AccessScope. Conversely, having participated in the AccessScope session first appeared necessary to answer questions regarding microscope operation correctly.

The quiz questions were almost identical for both methods, except the histological questions during the Digital slide test referred to a blood smear sample. A cross-section of stomach was used during the AccessScope test, in which the cell of interest in this slide was an epithelial cell. We believe differences in slide samples may have also contributed in test score differences, particularly in the group that participated in the AccessScope session first. The stomach section was comprised of more than one tissue type and several different organ structures. In addition, the cell of interest was only one of a few kinds of epithelial cells that were densely packed together. In contrast, the blood cells on the digital slide sample were loosely packed and consisted of only red blood cells, few platelets and fewer white blood cells, which all were round and differed only in colour and size. These cells were sparsely concentrated, which permitted them to be individually distinguishable. Reviewing the participants' scores for individual questions, it was questions pertaining to identifying cell shape and its function (questions 3, 4 and 5) that were answered the most correctly during the Digital slide test, especially after the second session.

We believe that the differences in training with the AccessScope system and the histological complexity in specimens likely played a role in test success. For future testing, we will devote more time training subjects in using AccessScope. We will also counterbalance slide samples during testing to control for differences in histology assessment. In addition, we will investigate using other quantitative tests that may be more sensitive to learning differences between active microscopy and reading digital histological images.

\section{Future development}

Design changes to the look of the GUI will be improved based on participants' suggestions, including adjusting font size, greater feedback and usability of certain controls and better description of commands for student users. We will also provide tracking of the user's present FOV location relative to the whole slide. Another future change to AccessScope would be to provide simultaneous control for multiple users through the Internet. By making AccessScope multi-use, one workstation could serve several students at the same time.

\section{Acknowledgements}

We are grateful to the participants of this study for their invaluable assistance. We appreciate Jennifer Danaher for helping with the participant trials and $\mathrm{Li}$ Hwa Chong for her assistance with participant evaluations. We also thank representatives from Optical Analysis Corp. and Olympus ${ }^{\circledR}$, Inc. for their help with hardware development and technical assistance. This project was made possible by a grant from the National Science Foundation (0533 124HRD to B.S.D.), financial support of the Center for Paralysis Research by the State of Indiana, and hardware and software support from Intel ${ }^{\circledR}$ QImaging ${ }^{\circledR}$ and Olympus ${ }^{\circledR}$, Inc.

\section{References}

1. Duerstock BS. Accessible microscopy workstation for students and scientists with mobility impairments. Asst Technol 2006;18:34-45.

2. Duerstock BS, Ahmed WM, Cirillo J, Robinson JP. AccessScope Project: accessible light microscopy for students with disabilities, In: Technology-based learning with disability (LWD-07). Dayton, OH: Wright State University; 2007. pp 30-36.

3. McCann WS. Science classrooms for students with special needs. Teaching strategies. J Early Edu Fam Rev 2000;7:2326.

4. Scruggs TE, Mastropieri MA. Refocusing microscope activities for special students. Sci Scope 1994;17:74-78.

5. Norman K, Caseau D, Stefanich GP. Teaching students with disabilities in inclusive science classrooms: Survey results. Sci Ed 1998;82:127-146.

6. Pence LE, Workman HJ, Riecke P. Effective laboratory experiences for students with disabilities: The role of a student laboratory assistant. J Chem Ed 2003;80:295-298. 
7. Mastropieri MA, Scruggs TE, Magnusen M. Activitiesoriented science instruction for students with disabilities. Learn Disabil Q 1999;22:240-249.

8. Salend SJ. Using an activities-based approach to teach science to students with disabilities. Interven School Clinic 1998;34: 67-72.

9. National Research Council. National science education standards.Washington, D.C.: National Academy Press; 1996.

10. Mastropieri MA, Scruggs TE, Mantzicopoulos P, Sturgeon A, Goodwin L, Chung S. "A place where living things affect and depend on each other": Qualitative and quantitative outcomes associated with inclusive science teaching. Sci Ed 1998;82: 163-179.

11. Michaels CA, Prezant FP, Morabito SM, Jackson K. Assistive and instructional technology for college students with disabilities: A national snapshot of postsecondary service providers. J Spec Educ Technol 2002;17:5-14.

12. Miner DL, Nieman R, Swanson AB, Woods M, editors. Teaching Chemistry to students with disabilities: A manual for high schools, colleges, and graduate programs. 4th ed. American Chemical Society Committee on Chemists with Disabilities. The American Chemical Society; 2001.

13. National Science Foundation, Division of Science Resources Statistics. S\&E doctorate recipients, by disability status: 2006, Survey of Earned Doctorates, 1999-2006. http://www.nsf.gov/ statistics/wmpd/pdf/tabf-13.pdf

14. National Science Foundation, Division of Science Resources Statistics. Employed scientists and engineers, by disability, status and age, Scientists and Engineers Statistical Data System. 2006. http://www.nsf.gov/statistics/wmpd/employ.cfm

15. Lau C, O'Leary S. Comparison of computer interface devices for persons with severe physical disabilities. Am J Occup Ther 1993;47:1022-1030.

\section{Appendix 1: AccessScope: User Performance Evaluation}

There are four types of tissues in the body: connective tissue, muscle tissue, nervous tissue and epithelium. Most organs or structures are made of more than one type of tissue. The brain, spinal cord and nerves are mainly composed of nervous tissue but also have some connective tissue. Likewise, skeletal and heart muscles are composed mostly of muscle tissue with some other tissues. Connective tissue includes ligaments, tendons, fat tissue, bones, blood, cartilage and other collagen-based structures. The last tissue type is epithelium. It composes the skin and the inside lining of cavities, such as blood vessels, the lungs, gastrointestinal tract and urinary tracts.

The function of cells with in these tissues can often be deduced based on their shape and their arrangement within a tissue or organ. For instance, cells of the nervous system play an important role in communication. The long processes or branches of these cells transmit electrical signals long distances within the brain and spinal cord and arranged in bundles. Muscle cells are closely linked and as- sembled in the same direction to contract as a group. Cells of connective tissue can either be densely compacted or spread apart in no apparent order. Their function is mainly structural in nature. Epithelial cells are usually regularly arranged in layers. These different layers have different functions such as lining cavities, secreting mucous, absorbing nutrients, or filtering particles.

Below are questions related to either slide A or B containing a section of organ or other structure composed of one or more of the four tissue types.

1. How was tissue B cut?
A. in lengthwise sections
B. in cross sections
C. unable to determine due to insufficient information

2. In slide $\mathrm{B}$, describe the different tissue regions at low magnification?

A. multiple colored regions arranged in defined layers

B. predominately one color in no apparent order

C. multiple colored regions in no apparent order but densely packed

D. multiple colored regions in no apparent order but loosely packed

3. In slide $\mathrm{B}$, what shape are the cells in this view?

A. round

B. stellate (having many branches)

C. long and thin

D. spindle-shaped

4. How do the shape and arrangement of the cells in slide B relate to their function?

A. round cells often secrete

B. long processes transmit electrical signals

C. these groups of cells arranged in a similar direction to perform a specific activity

D. a dense tangle of cells help maintain tissue structure and shape

5. What type of tissue is in slide B?
A. nervous
B. connective
C. muscle
D. epithelium

6. What part of the microscope controls magnification?
A. eyepieces
B. objectives
C. condenser
D. focus knob 